\title{
Computer-Aided Polymer Design: Integrating Group Contribution and Molecular Dynamics
}

Xinyuan Liang, , Xiang Zhang ${ }^{b}$, Lei Zhang ${ }^{a^{*}}$, Linlin Liu ${ }^{a}$, Jian Du ${ }^{a}$, Xiuling Zhuc , Ka Ming

$$
N g^{b}
$$

${ }^{a}$ Institute of Process Systems Engineering, School of Chemical Engineering, Dalian University of Technology, Dalian 116024, China

bDepartment of Chemical and Biomolecular Engineering, The Hong Kong University of Science and Technology, Clear Water Bay, Hong Kong

'State Key Lab of Fine Chemicals, Department of Polymer Science \& Materials, Dalian University of Technology, Dalian 116024, China

\footnotetext{
${ }^{*}$ Correspondence concerning this article should be addressed to Lei Zhang (keleiz@dlut.edu.cn).
}

\section{SUPPORTING INFORMATION}

Table S1. Additional structural constraints for polymer product design

$$
\begin{aligned}
& \text { For cyclic polymers: } \\
& n^{\max }(-q+1) \geq \sum_{i \in C Y C \cup A} n_{i} \\
& y \geqslant \frac{1}{M}(1-q) \\
& y \leqslant M(1-q) \\
& p_{1}=1-q+2 y \\
& \quad \sum_{i \in G_{2} \cap(C Y C \cup A)} n_{i} \geq y p_{1}
\end{aligned}
$$


$\sum_{i \in C Y C \cup A} n_{i} \geq y\left(v_{i_{1}}+1\right) \quad i_{1} \in C Y C \cup A$
$\sum_{i \in G_{3} \cap(C Y C \cup A)} n_{i} \geq y\left(-1.01 q+0.0001 \sum_{i_{1} \in C Y C \cup A} n_{i_{1}}\right)$
$3\left(\sum_{i \in G_{3} \cap(C Y C \cup A)} n_{i}-1\right)+\sum_{j \in G_{4} \cap(C Y C \cup A)} n_{j}+\sum_{k \in G_{2}} n_{\urcorner(C Y C \cup A)} n_{k} \geq 3-100(c$

For aromatic polymers:

$$
\begin{aligned}
& n_{A}=y_{1}(-q+1) \\
& y_{1} \leq \sum_{i \in A} n_{i} \\
& 6 n_{A}=\sum_{i \in A} n_{i} \\
& \sum_{i \in G_{3} \cap A} n_{i} \geq y\left(-1.01 q+0.0001 \sum_{i_{1} \in A} n_{i_{1}}\right) \\
& 3\left(\sum_{i \in G_{3} \cap A} n_{i}-1\right)+\sum_{j \in G_{4} \cap A} n_{j}+\sum_{\left.k \in G_{2} \cap\right\urcorner A} n_{k} \geq 3-100(q+1)
\end{aligned}
$$


Table S2. MD simulated data of the four properties of each system

\begin{tabular}{|c|c|c|c|c|c|c|}
\hline System & Structure & $C E D /(\mathrm{MPa})$ & $\begin{array}{c}\text { Average } \\
C E D /(\mathrm{MPa})\end{array}$ & $\rho /\left(\mathrm{kg} \cdot \mathrm{m}^{-3}\right)$ & $T_{g} /(\mathrm{K})$ & $\begin{array}{c}\kappa /\left(\mathrm{W} \cdot \mathrm{m}^{-1} \cdot \mathrm{K}^{-}\right. \\
1)\end{array}$ \\
\hline 1 & S1 & 260.8 & & 0.9272 & 168.8 & 0.3624 \\
\hline 2 & $\mathrm{~S} 1$ & 261.5 & 266.6 & 0.9563 & 191.4 & 0.3808 \\
\hline 3 & S1 & 277.4 & & 0.9637 & 201.7 & 0.4543 \\
\hline 4 & $\mathrm{~S} 2$ & 247.3 & & 0.8437 & 193.5 & 0.3574 \\
\hline 5 & $\mathrm{~S} 2$ & 259.1 & 254.2 & 0.8520 & 205.7 & 0.4093 \\
\hline 6 & S2 & 256.1 & & 0.8563 & 217.4 & 0.4940 \\
\hline 7 & S3 & 234.7 & & 0.8971 & 174.6 & 0.2375 \\
\hline 8 & $\mathrm{~S} 3$ & 239.8 & 236.8 & 0.9054 & 193.5 & 0.2584 \\
\hline 9 & S3 & 235.9 & & 0.9071 & 202.5 & 0.2624 \\
\hline
\end{tabular}




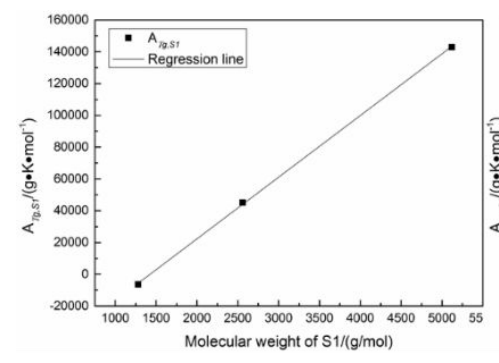

(a) Regression result of S1

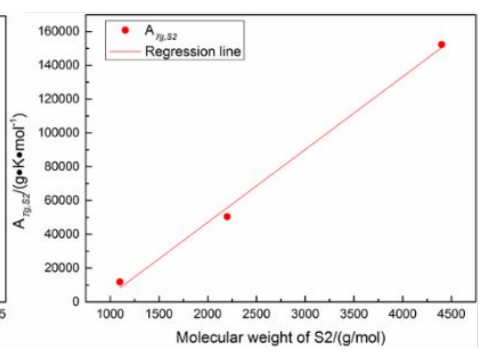

(b) Regression result of S2

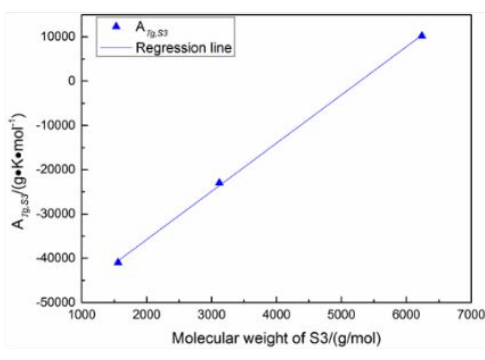

(c) Regression result of S3

Figure S1. Regression results of S1, S2 and S3 for $A_{T g, S}$ in the density estimation model

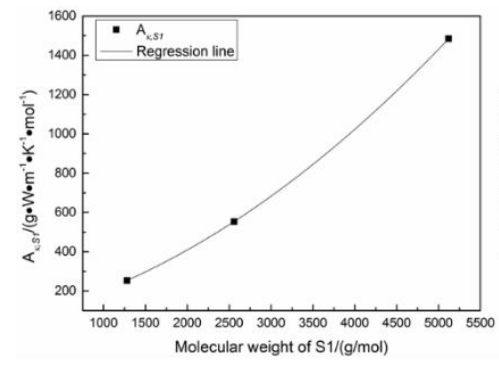

(a) Regression result of $\mathrm{S} 1$

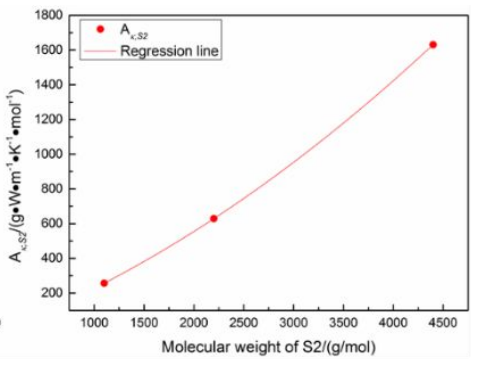

(b) Regression result of S2

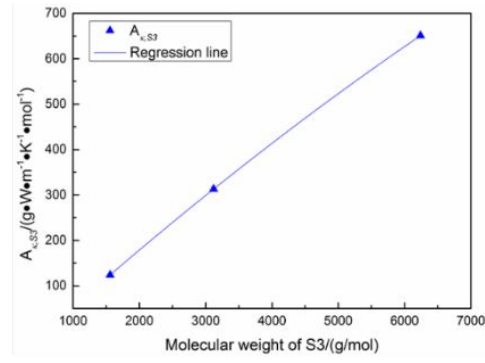

(c) Regression result of $\mathrm{S} 3$

Figure S2. Regression results of S1, S2 and S3 for $A_{\kappa, S}$ in the density estimation model

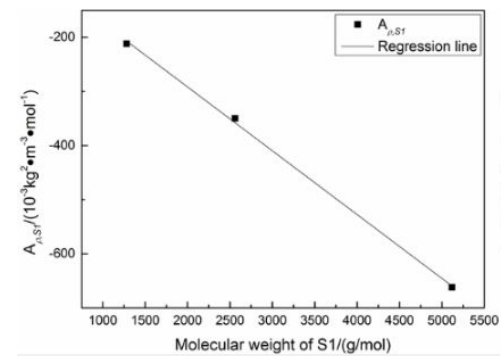

(a) Regression result of S1

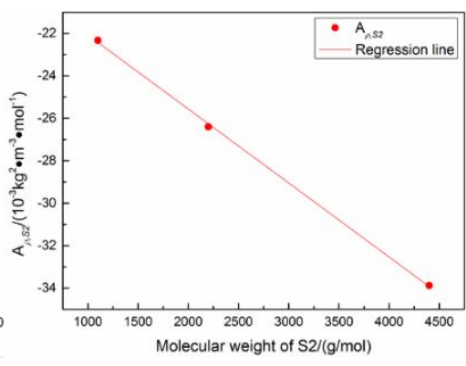

(b) Regression result of S2

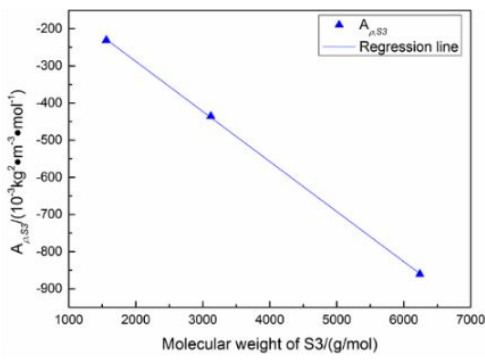

(c) Regression result of S3

Figure S3. Regression results of S1, S2 and S3 for $A_{\rho, S}$ in the density estimation model 